\title{
Desarrollo del nodo Venezuela en el campus virtual de salud pública
}

\author{
Malhi Cho, ${ }^{1}$ José Moya, ${ }^{1}$ Tulia Hernández, ${ }^{2}$ Maiqui Flores, ${ }^{3}$ Yelitza Ledezma, ${ }^{4}$ \\ Lenin Samán ${ }^{1}$ y Gabriel Listovsky
}

Forma de citar

Cho M, Moya J, Hernández T, Flores M, Ledezma Y, Samán L, Listovsky G. Desarrollo del nodo Venezuela en el campus virtual de la salud pública. Rev Panam Salud Publica. 2018;42:e71. https://doi. org/10.26633/RPSP.2018.71

RESUMEN El objetivo de este documento es describir los pasos en el desarrollo del nodo país del Campus Virtual de Salud Pública (CVSP) en Venezuela, para fortalecer la educación continua en entornos virtuales de aprendizaje, reforzar habilidades y conocimientos del personal de salud. Se definió como prioridad nacional la eliminación de barreras geográficas, ofrecer horarios flexibles y posibilidades de interacción en tiempo real con expertos a bajo costo fueron puntos resaltantes para el desarrollo del nodo en Venezuela. Los nodos del CVSP, tanto regionales como de países, que ofrece la Organización Panamericana de la Salud (OPS) permiten la formación masiva del personal de salud. Sin embargo, a pesar de algunas similitudes a nivel global y regional en temas de salud pública, los países tienen necesidades específicas según la situación epidemiológica, política, social y económica, y requieren dar respuestas a necesidades identificadas en el nivel local. En Venezuela, el enfoque del fortalecimiento en las competencias y la formación continua del personal de salud se orienta hacia los 13921 equipos básicos de salud desplegados en los 24 estados del país. Ante esta realidad, la oficina nacional de la OPS apoyó el desarrollo del CVSP nodo Venezuela.

Palabras clave

Educación continua; calidad de la atención de salud; atención primaria de salud; recursos humanos; Venezuela.

En el avance de los compromisos asumidos por los países de la Región de las Américas para el acceso universal a la salud y la cobertura universal de salud según resolución CD 53/5 de 2014 (1) y el Objetivo 3 de la Agenda 2030 para el

\footnotetext{
Organización Panamericana de la Salud, Caracas, Venezuela. Enviar correspondencia a Malhi Cho, chomalhi@paho.org

2 Universidad de Ciencias de la Salud, Caracas, Venezuela

3 Ministerio del Poder Popular para la Salud, Caracas, Venezuela.
}

Desarrollo Sostenible (2), uno de los pilares para alcanzar las metas establecidas es la formación del personal de salud, sean profesionales, técnicos o de nuevos perfiles, en coherencia con los cambios en el modelo de atención propuestos por

\footnotetext{
4 Instituto de Altos Estudios “Dr. Arnoldo Gabaldón", Maracay, Venezuela.

5 Organización Panamericana de la Salud, Panamá, Panamá.
}

Venezuela para el logro de la Salud Universal.

Para lograr la salud universal se requiere de recursos humanos en cantidad suficiente, distribuidos equitativamente y con capacidades adecuadas, según las necesidades de las comunidades (3). Estos recursos deben desarrollar, mediante la formación, un perfil con aptitudes y actitudes que les permita ofrecer servicios de calidad basados en el modelo de salud definido por el país. Alcanzar esta meta en recursos humanos con énfasis en 
la atención primaria en salud (APS) es aún una agenda pendiente para muchos países de la Región que trabajan en la conformación de equipos de salud con grupos multiprofesionales capacitados para dar respuestas a las necesidades de salud de la población. Los sistemas de salud deben desarrollar las capacidades resolutivas en la APS mediante el fortalecimiento de los servicios en infraestructura, equipamiento e insumos, así como el fortalecimiento de los grupos de salud multiprofesionales mediante el desarrollo de competencias a través de la educación continuada, con el objeto de que los conocimientos recibidos de manera oportuna y actualizada les permita responder con eficiencia y calidad a los requerimientos en salud de la población. En vista de la trascendencia del tema, la Organización Panamericana de la Salud (OPS) presentó la estrategia sobre las políticas nacionales de recursos humanos para la salud aprobados en la Resolución CSP29.R15 (3).

La OPS, sensible a esta realidad en los países de la Región, propone en su estrategia de salud universal trabajar en la formación de los recursos humanos para ampliar el acceso y la cobertura con equidad y calidad mediante la concertación con el sector educativo, alineando las estrategias de formación bajo un nuevo paradigma que dé respuesta a las necesidades de los sistemas de salud y al desarrollo de políticas de educación continua mediante la diversificación de las metodologías, la incorporación de la educación virtual y la innovación en el uso de las tecnologías para acompañar los procesos de cambio hacia el acceso universal y la cobertura universal de salud (1). La educación permanente debe dirigirse a reducir las brechas de conocimiento y permitir la construcción de un sistema de aprendizaje que apoye el desarrollo de habilidades y promueva las competencias técnicas, programáticas, gerenciales y administrativas.

En la República Bolivariana de Venezuela, se definió la estrategia para el fortalecimiento de la APS mediante la Misión Barrio Adentro desde 2003. Como parte de esta estrategia, se inicia en 2005 la formación de médicos integrales comunitarios (MIC), enfermeros integrales comunitarios y promotores de salud, a fin de aumentar la disponibilidad de profesionales de la salud con un perfil orientado a la atención comunitaria y conformar los EBS de la red de atención comunal de salud (RACS).
En 2011 egresó la primera cohorte de MIC y, para diciembre de 2016, habían egresado $20241 \mathrm{MIC}$ en cinco cohortes. Los profesionales graduados fueron contratados por el Ministerio del Poder Popular para la Salud (MPPS) y se encuentran distribuidos a nivel nacional como parte de los EBS de los consultorios populares ${ }^{6}$, donde brindan asistencia sanitaria basada en APS en los 24 estados del país. Estos consultorios constituyen escenarios docente-asistenciales, cuyos EBS conformados por 13921 profesionales de la medicina (médicos integrales comunitarios, médicos generales, especialistas en medicina general integral y especialistas en medicina familiar), 8705 profesionales de enfermería (auxiliares de enfermería, técnicos superiores universitarios en enfermería y licenciados en enfermería) y 6139 promotores de la salud (auxiliares de medicina simplificada, agentes comunitarios de atención primaria de salud, defensores de salud, promotores de salud y trabajadores sociales) son los encargados de realizar o ejecutar actividades y desarrollar competencias, de acuerdo a las áreas de intervención, en materia de promoción, prevención de riesgos, enfermedades y otros daños a la salud, atención integral, rehabilitación, higiene sanitaria, docencia e investigación, así como otros aspectos que el MPPS considere necesarios (5).

Para definir y coordinar la agenda de formación continua del personal de salud, se requiere mantener una articulación fluida entre el MPPS como ente rector que determina las políticas de formación en materia de salud y las instituciones educativas adscritas a este, que ejecutan los programas de formación y actualización para el personal del sistema público de salud, lo que ayuda a minimizar las barreras de acceso. El objetivo es asegurar que la oferta de formación continua corresponda con las competencias necesarias y requeridas para ofrecer una atención integral y resolutiva del sistema de salud a la población y que la capacitación, información y educación permitan incrementar y fortalecer la masa crítica de recursos humanos en salud para

\footnotetext{
El consultorio popular es el establecimiento de salud del Sistema Público Nacional de Salud que constituye la unidad fundamental y primordial a partir de la cual se coordinan las acciones del modelo de atención integral a las personas, las familias, la comunidad, el medio ambiente, el medio laboral y el medio escolar, entre otros (4).
}

ofrecer calidad en la atención y la mejora continua.

Los cambios acelerados en los perfiles epidemiológicos y la necesidad de socializar de forma masiva nuevos conocimientos hacen inminente la búsqueda de metodologías que permitan alcanzar los objetivos de elevar las competencias y las capacidades en forma continua y dinámica, y así apoyar al personal de la salud que se encuentra distribuido en todo el país para lograr la prestación de la atención a la salud con calidad.

\section{Campus virtual de salud pública}

El aprendizaje y los intercambios entre las personas se realizan de manera permanente a lo largo de la vida mediante ámbitos y fuentes de conocimiento que incluyen desde los ambientes educativos, de trabajo, en grupos sociales, bibliotecas, redes de información, listas de interés y en todo el acervo social (6). La expansión del conocimiento y las tecnologías de la información y comunicación (TIC) han ampliado en forma sustantiva los espacios para aprender de y con los otros. El uso de las TIC en educación supera barreras geográficas y temporales entre el educador y educando; propicia el uso de los medios tecnológicos disponibles; potencia la gestión del conocimiento; mejora el desempeño de programas de educación permanente y la interacción entre personas y grupos de diferentes contextos (7), lo que reivindica la íntima relación que existe entre el aprendizaje, la generación de conocimiento, la innovación continua y el uso de las TIC (8).

Para dar respuesta a necesidades de metodologías dinámicas y versátiles para la masificación de los conocimientos, la OPS incorporó la formación en entornos virtuales y desarrolló el CVSP, el cual dispone de un espacio de trabajo en red de personas, instituciones y organizaciones que comparten cursos, recursos, servicios y actividades de educación e información, con el propósito de mejorar las competencias de la fuerza laboral y las prácticas de la salud pública (9). El CVSP ofrece la posibilidad de ampliar la oferta académica, con las ventajas descritas sobre el uso de las TIC y aporta disponibilidad de nuevos entornos de aprendizaje; esto permite compartir conocimientos, crear contenidos para el proceso de enseñanzaaprendizaje, intercambiar información y 
fomentar procesos de comunicación y construcción de aprendizaje (7). De esta manera, la herramienta permite potenciar la gestión del conocimiento y los programas de educación continua para los recursos humanos y las instituciones en salud pública mediante el uso de las TIC para la mejora continua en el desempeño de las prácticas de la salud y de los programas de educación en salud, a la vez que favorece la autonomía del estudiante y mejora la capacidad de comunicarse de forma escrita por medio del buen uso del lenguaje (10).

La concepción del Campus Virtual de Salud Pública de la OPS se realizó a partir de ideas y experiencias acumuladas y lanzadas en el año 2000. En 2005, la directora de la OPS reafirmó, en la $4^{\text {a }}$ Reunión de Coordinación Regional de la Biblioteca Virtual de Salud en Brasil, el compromiso para la construcción del CVSP, al cual se irían sumando, de manera progresiva, redes de iniciativas y experiencias internacionales, regionales y nacionales para la educación continua de los trabajadores de salud. Entre el 2009 y el 2010, se pone en marcha la iniciativa a nivel regional y se conforman los primeros nodos en los países.

Esta metodología de difusión de conocimientos tuvo una aceptación masiva: se pasó de 174568 matriculados en enero de 2016 a 394111 matriculados en diciembre de 2017, con un incremento de aproximadamente 10000 usuarios cada mes. El CVSP disponía, para mediados de 2017, de 203 cursos entre tutorados y de autoaprendizaje, de los cuales 34 cuentan con más de 1000 participantes cada uno (11).

Para que se instituya una formación continua que responda a las prioridades, las instituciones de salud pública de los países deben identificar las necesidades de cursos, actividades educativas y de información que permitan fortalecer al sistema de salud mediante el uso de metodologías innovadoras y las TIC como herramientas valiosas para la descentralización y el incremento en la cobertura de los procesos educativos para contribuir al fortalecimiento institucional y de la fuerza laboral.

El CVSP está constituido por un nodo regional, nodos países y nodos institucionales en los países. Estos constituyen espacios en el que las áreas, las unidades, los programas y los proyectos de una institución o de un conjunto de instituciones y organizaciones colaboran de manera activa en red y comparten recursos educativos en salud pública (12).

\section{CVSP-Venezuela}

En Venezuela, el número de establecimientos de la RACS se ha incrementado $72 \%$ en los últimos 15 años (13); para el año 2017, contaba con 13921 EBS que requieren de formación continua mediante cursos de actualización. El país se involucró en el proceso de desarrollo del CVSP desde el 2010, cuando se propuso al Instituto de Altos Estudios (IAE) “Dr. Arnoldo Gabaldón", en la ciudad de Maracay, la difusión de los cursos regionales. Durante el período de consolidación regional de las redes del CVSP, se recibió en Venezuela la visita del coordinador regional en julio de 2013. En ese momento, la OPS propuso desarrollar el nodo de Venezuela, dadas las características de avance del país en el tema de las TIC y las necesidades manifestadas de requerimientos de formación continua. El IAE, responsable de los posgrados de salud pública, asumió el liderazgo en esta iniciativa luego de analizar la utilidad que la metodología podría ofrecer al contexto país; como entidad adscrita al MPPS presentó el informe y solicitó los permisos requeridos para la creación del Nodo Venezuela. Posteriormente, se realizaron múltiples encuentros virtuales y presenciales entre los actores locales y el nivel regional con el objeto de articular un plan para la implementación del CVSP-Nodo en Venezuela (CVSP-VEN).

El país analizó las potencialidades de la metodología y decidió utilizar el CVSP con el apoyo de la OPS de Venezuela, como una herramienta de formación masiva en salud pública. Para ello, se constituyó un grupo de trabajo integrado por el MPPS y sus entes adscritos, cuya misión es la ejecución de las políticas de formación de recursos humanos en salud a nivel de pregrado, posgrado y formación continua. Al inicio, participaron el IAE e Instituto Nacional de Higiene "Rafael Rangel" (INHRR), luego se incorporó, tras su creación (14), la Universidad de Ciencias de la Salud "Hugo Chávez Frías" (UCS). La participación de la UCS fue considerada esencial, ya que es la única universidad nacional adscrita al MPPS que forma parte del Sistema Público Nacional de Salud y está sujeta a las políticas y lineamientos del MPPS, además del Sistema de Educación Universitaria.

A pesar del entusiasmo inicial, las acciones se vieron enlentecidas en el proceso para el desarrollo del CVSP-VEN por razones políticas y técnicas. Entre las primeras se hallan los cambios de gestión y de prioridades de las autoridades, así como una decisión política del momento para desarrollar y albergar la plataforma país con infraestructura, equipamiento y capacidad de funcionamiento autónomos, como un instrumento de empoderamiento nacional.

Dentro de las razones técnicas, el INHRR disponía de la infraestructura de tecnología de información (TI) de última generación para esta función; sin embargo, faltaba garantizar un ancho de banda suficiente para atender la demanda real y proyectada del nodo, así como desarrollar un esquema de funcionamiento de alta disponibilidad que le permitiera operatividad continua en caso de fallos; es decir, una infraestructura paralela de respaldo.

Se realizaron encuentros durante el 2014 sin alcanzar los resultados esperados; en el año 2015 se vuelve a presentar la propuesta a las nuevas autoridades de salud, reconsiderando la metodología y las herramientas para la formación continua a las cohortes de medicina integral comunitaria, enfermería integral comunitaria y promotores sociales de salud desplegados a nivel nacional en los consultorios populares.

Como parte del proceso para el nodo país, en el año 2016 se elevó una solicitud a la ministra de salud, en la que se pedía autorización para crear el nodo de Venezuela y utilizar la plataforma del CVSP de la OPS hasta que la infraestructura nacional fuera adecuada para la migración de los servicios de aulas virtuales, conferencias web y repositorios de recursos educativos abiertos (REA).

Se elaboró, también, un documento de acuerdo entre el MPPS y la OPS sobre la cooperación para la gestión de cursos y recursos educativos en el CVSP-VEN el cual, tras revisiones de las oficinas de legales correspondientes, fue firmado por ambas partes con aprobación final en abril de 2017 (15).

Para compartir información del CVSP y su utilización, se organizó el taller "Desarrollo de los Nodos del CVSP", en el cual participó el coordinador regional del CVSP, quien mantuvo reuniones con diversas instancias del MPPS; entre estas, la Oficina de Integración y Asuntos Internacionales (OIAI), que expuso la visión, misión y objetivos del CVSP, experiencias y avances en los diferentes países con el desarrollo de los nodos; así como las medidas de seguridad en 
el manejo de la información al utilizar el espacio virtual en el desarrollo de cursos de formación para cada país.

Mientras el proceso de formalización del CVSP-VEN seguía su curso, se decidió avanzar en la formación de tutores y coordinadores de futuros cursos y se organizó el primer curso como CVSP-VEN mediante el uso de la plataforma de nivel regional.

Antes de este curso, se desarrolló en 2015 un curso nacional sobre "Metodología de Gestión Productiva de Servicios de Salud" para participantes de Venezuela, elaborado y tutorado por consultores internacionales (16). El curso se dictó bajo la modalidad semipresencial contando con 27 participantes procedentes de los 24 estados del país, de los cuales 18 (75\%) culminaron en forma exitosa. La selección de participantes fue realizada por el MPPS con el propósito de obtener propuestas de intervención para las instituciones de los estados que representaban.

Una vez formalizado el acuerdo del CVSP-VEN, se realizaron reuniones sucesivas en las que se plasmaron los términos de la cooperación para la creación del CVSP-VEN y la implementación de los cursos. Se destacó, en primer lugar, que la coordinación o Secretaría debe ser rotatoria con una duración de dos años, con base en la elección realizada entre las instituciones que lo conforman y, en segundo lugar, que se debían establecer el comité de dirección y sus comisiones de trabajo: comité de gobernanza, comisión académica y comisión técnica.

El comité de gobernanza es responsable de los aspectos operativos, administrativos y financieros de los cursos gestionados por la Secretaría, aprobar los contenidos de los cursos y recursos de aprendizaje (CRA) que se incorporen al CVSP-VEN cumpliendo las normas del CVSP y, de esta manera, aseguren la calidad y las licencias requeridas para impartirlos. Este comité es responsable, también, de identificar necesidades de capacitación según las prioridades nacionales de salud pública, entre otras funciones. La OPS/OMS forma parte del Comité de Gobernanza del CVSP-VEN a través de la Oficina país OPS-VEN y bajo asesoría del CVSP regional para brindar acceso a los componentes tecnológicos. Además, presta asesoría al CVSP-VEN en temas de políticas y estrategias para que su funcionamiento esté alineado con las directrices establecidas en el acuerdo de cooperación de la OPS/OMS, a fin de apoyar al fortalecimiento de capacidades y competencias de los profesionales de salud para atender los problemas de salud pública, atención médica y fortalecimiento de los procesos gerenciales del sector salud.

La Secretaría inicial quedó bajo el liderazgo del MPPS; el comité de gobernanza con sus comisiones inició la elaboración de la norma que regula la formación en salud pública en entornos virtuales de aprendizaje y su propuesta de resolución ministerial, actualmente en revisión por la consultoría jurídica del MPPS. Este es el marco normativo y de funcionamiento que regirá el CVSP-VEN en lo administrativo, académico, técnico y de seguimiento y calidad de los recursos educativos. La comisión técnica garantiza la capacitación, desarrollo y funcionamiento de la infraestructura de redes de información, telecomunicación y operacionalización del campus desde el punto de vista tecnológico y metodológico en el ámbito nacional.

La Comisión académica es la encargada de evaluar la calidad y pertinencia de los recursos educativos en el CVSP-VEN y formula las recomendaciones correspondientes al comité de gobernanza. Además, gestiona con expertos la creación, revisión de contenidos y estructuras de los cursos y recursos de aprendizaje. Es conocido el desafío que representa el desarrollo de contenidos de calidad para la formación a distancia (17); por esta razón, la primera necesidad identificada para el CVSP-VEN fue la formación de un grupo de profesionales, expertos en sus correspondientes áreas, con conocimientos y destrezas para desarrollar cursos virtuales y capacidad de coordinar y tutorar los cursos en formato virtual, con la finalidad de fortalecer la capacidad local de elaboración de cursos según requerimientos y prioridades del país. Para tal efecto, se solicitó la migración del Curso Regional de Tutores, Edición 2016. En la revisión de los contenidos, se consideró la importancia de incorporar un módulo adicional, que se denominó "Módulo 0", correspondiente al marco político conceptual del Sistema Público Nacional de Salud de la República Bolivariana de Venezuela.

Para la primera edición del curso de tutores, que se desarrolló entre el 21 de abril y el 27 de setiembre de 2017, se convocó a 220 participantes por las instituciones que conforman el CVSP-VEN. Se recibieron 117 cartas compromiso. El "Módulo 0" lo iniciaron 114 participantes; sin embargo, el curso fue suspendido por aproximadamente un mes debido a razones externas al CVSP-VEN. Esta situación ocasionó una baja de 26 participantes, por lo que al inicio del Módulo I había 88 personas, de las cuales 51 (58\%), procedentes de nueve estados del país, egresaron con éxito.

Se identificaron áreas prioritarias para la elaboración de cursos virtuales en el corto plazo, entre los cuales se encuentran: dirección y gestión de las áreas de salud integral comunitarias (ASIC), curso básico de epidemiología, gestión y uso racional de medicamentos y una guía práctica sobre programas de salud priorizados para el equipo básico de salud en la red de atención comunal (18). Se espera que estos cursos estén listos para su inicio en 2018 y se proyecta un total de 60000 personas beneficiados de la formación continua.

\section{REFLEXIONES}

Para lograr la mejora en la calidad de la atención en salud, se requiere fortalecer habilidades y conocimientos de los equipos básicos de salud a nivel nacional, por lo que es prioritario el desarrollo de cursos que permitan, mediante la superación de barreras geográficas y temporales, la formación continua.

En Venezuela, el CVSP-VEN ha permitido incorporar un modelo de educación continua para los EBS que permite el fortalecimiento de competencias $y$ habilidades.

El CVSP-VEN se utiliza para dar respuesta a las necesidades de capacitación ante situaciones sanitarias específicas del país.

Si bien es una metodología que está demostrando sus potencialidades, aún existen tensiones con los defensores de la educación tradicional presencial, además de la necesidad de asegurar la conectividad a nivel nacional y que los participantes tengan conocimientos básicos en informática.

Un desafío presente es el desarrollo de contenidos de calidad para los cursos a distancia, con la rapidez requerida por el país.

Conflicto de intereses. Ninguno declarado por los autores.

Declaración. Las opiniones expresadas en este manuscrito son responsabilidad de los autores y no reflejan necesariamente los criterios ni la política de la RPSP/PAJPH o de la Organización Panamericana de la Salud. 


\section{REFERENCIAS}

1. Organización Panamericana de la Salud. Estrategia para el Acceso Universal a la Salud y la Cobertura Universal de la Salud; 2014.

2. Naciones Unidas. Agenda 2030 y Los Objetivos de Desarrollo Sostenible. Disponible en: http://www.un.org/sustainabledevelopment/es/objetivos-dedesarrollo-sostenible.

3. Organización Panamericana de la Salud (OPS). Estrategia de Recursos Humanos para el Acceso Universal a la Salud y la Cobertura Universal de la Salud. Informe No. CSP29/10. Washington D.C.: OPS; 2017.

4. Ministerio del Poder Popular para la Salud de la República Bolivariana de Venezuela. Manual de Trabajo del Equipo Básico de Salud del Consultorio Popular. Caracas: Organización Panamericana de la Salud; 2017.

5. Ministerio del Poder Popular para la Salud de la República Bolivariana de Venezuela. Datos no publicados, Viceministerio de Redes de Atención Ambulatoria de Salud del MPPS; 2016.n

6. Organización Panamericana de la Salud. Campus Virtual de Salud Pública. Glosario General. Consultado en noviembre de 2017. Disponible en: https://cursos.campusvirtualsp.org/mod/glossary/view. php?id=1242\&mode=letter\&hook=A\&sortkey $=\&$ sortorder $=$

7. Tarasow, F. ¿De la educación a distancia a la educación en línea? ¿Continuidad o comienzo? en diseño de intervenciones educativas en línea, Carrera de Especialización en Educación y Nuevas Tecnologías. Proyecto Educación en Nuevas TecnologíasFacultad Latinoamericana de Ciencias Sociales (PENT-FLACSO) Argentina. Módulo: Diseño de intervenciones educativas en línea; 2010. Disponible en: http://www.pent.org.ar/institucional/publicaciones/educacion-distancia-educacionlinea-continuidad-comienzo

8. Granados JJ, Fernández Rodríguez JC, Lombardero Rodil L. Formación de postgrado con metodología e-learning. Revista Iberoamericana para la Investigación y el Desarrollo Educativo 2013;10:57-69.

9. Organización Panamericana de la Salud. Campus Virtual de Salud Pública. Disponible en: https://www.campusvirtualsp.org/es/que-es-el-campus

10. Gil Rendón ME, Gallardo Córdova KE. Análisis del proceso de acompañamiento en la trayectoria de formación a nivel posgrado en un programa en línea. XIV Congreso Nacional de Investigación Educativa. San Luis de Potosí: Consejo Mexicano de Investigación educativa; 2017. Disponible en http://www.comie.org.mx/congreso/ memoriaelectronica/v14/doc/1944.pdf

11. Organización Panamericana de la Salud. Campus Virtual de Salud Pública. TableauPublic. Disponible en: https://public. tableau.com/profile/campusvirtualsp\#!/ vizhome/cvsp/UsuariosdelCVSP

12. Organización Panamericana de la Salud. Modelo de Gobernanza del Campus Virtual de Salud Pública. Disponible en https: / / www.campusvirtualsp.org / sites/default/files/download/modelos/ GobernanzaCVSP_08.pdf

13. Instituto Nacional de Estadística de la República Bolivariana de Venezuela. Censo 2001. Caracas: Ministerio de Planificación y Desarrollo; 2001.

14. República Bolivariana de Venezuela. Presidencia de la República. Decreto de creación de la Universidad de las Ciencias de la Salud (8 de octubre de 2014). En:
Tribunal Supremo de Justicia. Gaceta Oficial de la República, 40.514.

15. Ministerio del Poder Popular para la Salud de la República Bolivariana de Venezuela (MPPS) y Organización Panamericana de la Salud (OPS). Administración de cursos y recursos educativos. Acuerdo de Cooperación Técnica entre el MPPS de la República Bolivariana de Venezuela y la OPS/OMS. Caracas; 2017.

16. Organización Panamericana de la Salud. Campus Virtual de Salud Pública. Curso de Metodología de Getión Productiva de Servicios de Salud, Edición Venezuela 2015. Disponible en: https:/ / cursospaises. campusvirtualsp.org/course/index. php? categoryid $=54$

17. Pérez SC, Facchini HA. El diseño instruccional y la bimodalidad en las carreras de posgrado: experiencia en la UTN (2016). Mendoza, Argentina. Disponible en: https: / / www.researchgate.net / publication /311576604_El_diseno_ instruccional_y_la_bimodalidad_en_las_ Carreras_de_Posgrado_Experiencia_en_ la_UTN

18. Ministerio del Poder Popular para la Salud de la República Bolivariana de Venezuela. Guía práctica sobre problemas priorizados para el equipo básico de salud en la red de atención comunal. Caracas: Organización Panamericana de la Salud; 2017.

Manuscrito recibido el 7 de diciembre de 2017. Aceptado para su publicación, tras revisión, el 6 de marzo de 2018.

ABSTRACT The objective of this document is to describe the steps in the development of the country node of the Virtual Campus for Public Health (VPHC) in Venezuela, with a view to enhancing continuing education in virtual learning environments and strengthening the abilities and knowledge of health workers, which was defined as a national priority. Eliminating geographical barriers, offering flexible schedules, and providing opportunities for real-time, low-cost interaction with experts were key aspects of the development of the Venezuela node. The VPHC nodes offered by the Pan American Health Organization (PAHO), both at the regional and country levels, allow for large-scale training of health workers. However, despite similarities in public health issues at the global and regional levels, each country has specific needs according to its epidemiological, political, social, and economic situation, and has to respond to needs identified at the local level. In Venezuela, the focus is on strengthening competencies and providing continuing education to the 13921 basic health teams deployed in the country's 24 states. It was in this context that the PAHO country office supported the development of the Venezuela node of the VPHC.

Keywords Education, continuing; quality of health care; primary health care; human resources; Venezuela. 
RESUMO O objetivo desta análise é descrever os passos no desenvolvimento do núcleo país do Campus Virtual de Saúde Pública (CVSP) na Venezuela. Incentivar a educação contínua em espaços virtuais de aprendizagem, reforçar habilidades e conhecimentos dos

Desenvolvimento do núcleo Venezuela no Campus Virtual de Saúde Pública

Palavras-chave profissionais da saúde definido como prioridade nacional, eliminar barreiras geográficas e proporcionar horários flexíveis e a possibilidade de interação em tempo real com especialistas a um baixo custo foram aspectos destacados para o desenvolvimento do núcleo na Venezuela. Os núcleos do CVSP, tanto regionais como nos países, propiciados pela Organização Pan-Americana da Saúde (OPAS), permitem a formação em massa de profissionais da saúde. No entanto, apesar das semelhanças em saúde pública nas esferas global e regional, os países têm necessidades próprias de acordo com a situação epidemiológica, política, social e econômica e precisam atender as necessidades identificadas ao nível local. Na Venezuela, o enfoque de fortalecimento das competências e a formação contínua dos profissionais da saúde é direcionado às 13921 equipes básicas de saúde implantadas nos 24 estados do país. Diante desta realidade, a repartição da OPAS no país deu apoio ao desenvolvimento do núcleo Venezuela do CVSP.

Educação continuada; qualidade da assistência à saúde; atenção primária à saúde; recursos humanos; Venezuela. 